\title{
Suppressive Effects of Plumbagin on Invasion and Migration of Breast Cancer Cells via the Inhibition of STAT3 Signa ling and Down-regulation of Inflammatory Cytokine Expressions
}

\author{
Wei Yan ${ }^{1,2}$, Bing Tu${ }^{1}$, Yun-yun Liu³, Ting-yu Wang4, Han Qiao' ${ }^{1}$ Zan-jing Zhai ${ }^{1}$, Hao-wei Li ${ }^{1}$, Ting-ting Tang ${ }^{*}$ \\ ${ }^{1}$ Shanghai Key Laboratory of Orthopaedic Implants, Department of Orthopaedic Surgery, Shanghai Ninth People's Hospital, \\ Shanghai Jiao Tong University School of Medicine, Shanghai 200011, China; ${ }^{2}$ Wendeng Zhenggu Hospital of Shandong \\ Province, Wendeng, Shandong 26440o, China; 3Department of Gynecology and Obstetrics, Wendeng Center Hospital of \\ Weihai City, Weihai, Shandong 26440o, China; 4Department of Pharmacy, Shanghai Ninth People's Hospital, Shanghai Jiao \\ Tong University School of Medicine, Shanghai 200011, China
}

Objective: The aim of this study was to investigate the effects of plumbagin (PL), a naphthoquinone derived from the medicinal plant plumbago zeylanica, on the invasion and migration of human breast cancer cells. Methods: Human breast cancer MDA-MB-231SArfp cells were treated with different concentrations of plumbagin for $24 \mathrm{~h}$. The effects of plumbagin on the migration and invasion were observed by a transwell method. The expressions of IL-1a, IL-1 $\beta$, IL-6, IL-8, TGF- $\beta$, TNFa, MMP-2 and MMP-9 mRNA in MDA-MB-231SArfp cells were detected using Real-Time PCR. MDA-MB-231SArfp cells were treated with plumbagin at different concentrations for 45 minutes. The activation of STAT3 was detected by western blot. Following this analysis, STAT3 in MDA-MB-231SArfp cells was knocked out using specific siRNA. mRNA levels of IL-1a, TGF- $\beta$, MMP-2 and MMP-9 were then detected. Consequently, MDA-MB-231SArfp cells were injected intracardially into BALB/c nude mice to construct a breast cancer bone metastatic model. The mice were injected intraperitoneally with plumbagin. Non-invasive in vivo monitoring, $X$-ray imaging and histological staining were performed to investigate the effects of plumbagin on the invasion and migration of breast cancer cells in vivo. Results: The in vitro results showed that plumbagin could suppress the migration and invasion of breast cancer cells and down-regulate mRNA expressions of IL-1a, TGF- $\beta$, MMP-2 and MMP-9. Western blotting demonstrated that plumbagin inhibited the activation of STAT3 signaling in MDA-MB-231SArfp cells. The inactivation of STAT3 was found to have an inhibitory effect on the expressions of IL-1a, TGF- $\beta$, MMP-2 and MMP-9. In vivo studies showed that plumbagin inhibited the metastasis of breast cancer cells and decreased osteolytic bone metastases, as well as the secretion of MMP-2 and MMP-9 by tumor cells at metastatic lesions. Conclusions: Plumbagin can suppress the invasion and migration of breast cancer cells via the inhibition of STAT3 signaling and by downregulation of IL-1a, TGF- $\beta$, MMP-2 and MMP-9.

Keywords: plumbagin; breast cancer; invasion; migration

Bone Research (2013) 4: 362-370. doi: 10.4248/BR201304007

\section{Introduction}

Breast cancer is the most common cancer in women,

*Correspondence: Ting-ting Tang

E-mail: tingtingtang@hotmail.com

Received 09 September 2013; Acc epted 17 October 2013 and bone tissues are the most frequent sites for metastases. Up to $70 \%$ of patients with metastatic breast cancer develop bone metastases that induce increased osteoclast activity, resulting in local bone destruction and skeletal complications, including pain, hypercalcemia, and nerve compression (1-3). The development and outgrowth of these secondary lesions rely upon the 
intric ate cellular and molecular interactions between mammary tumor cells in the bone microenvironment. Tumor cells secrete signaling proteins, such as parathyroid homone-related peptide (PTHrP) (4), to promote the sec retion of RANKL, the receptor activator of nuclear factor-kB ligand, in osteoblast cells that activates bone osteolysis induced by osteoclasts. The concomitant bone destruction releases various growth factors, including transforming growth factor- $\beta$ (TGF- $\beta$ ) and insulin-like growth factor-1 (IGF-1), to further bind the receptors on the surface of tumor cells and enhance cell proliferation and PTHrP production through activation of the Smad/ MAPK signa ling transduction pathways, thus establishing a vicious cycle of bone metastases (5). The most widely used treatments in skeletal c omplic ations of varied neoplastic diseases are bisphosphonates (BPs) that block osteoc last ac tivity; this application has been effective in decreasing fomation of bone lesions, although this strategy fails to induce the bone regeneration (6). Furthermore, a growing number of case reports have demonstrated that long-term BP therapy might lead to osteonecrosis of the jaw (ONJ) (7-10). Therefore, the search for treatment options that can effectively inhibit tumor growth and reduce bone destruction caused by tumor metastases with mitigated side effects is of pivotal significance.

Plumbagin (5-hydroxy-2-methyl-1,4-naphthoquinone), derived from the roots of the medical plant plumbago zeylanica, is one of the most investigated compounds. Recent studies have reported the antibacterial effects (11), anti-inflammatory effects (12), and anticancer activities of plumbagin both in vitro (13) and in vivo (1415). Previous studies have shown that plumbagin can inhibit tumor cell proliferation in vitro by inducing apoptosis and autophagy of breast neoplasm cells (13) and the invasion of prosta te cancer cells (14). Recent studies have also demonstrated that plumbagin can inhibits osteoc lastogenesis and reduces human breast cancerinduced osteolytic bone metastasis (16-18), however, more in vivo evidence need to be supplemented and the relevant mechanisms still remain to be investigated. Therefore, the aim of this study is to investigate whether there are any suppressive effects of plumbagin on the invasion and migration of human breast cancer MDAMB-231SArfp cells and to explore potential functional mechanisms. We thus established an animal model of breast cancer bone metastases via injection of breast cancer cells intracardially. A non-invasive small animal monitoring system in addition to X-ray imaging and histological analysis were employed to monitor the curative effects of plumbag in on breast cancerbone metastases in real time.

\section{Materials and Methods}

\section{Main reagents}

Plumbagin and dimethyl sulfoxides (DMSO) were purchased from Sigma-Aldrich (St. Louis, MO, USA). For cell culture experiments, plumbagin was dissolved in DMSO at a concentration of $200 \mathrm{mmol} \cdot \mathrm{L}^{-1}$ and stored in a dark bottle at $-20{ }^{\circ} \mathrm{C}$. This stock solution was diluted further in cell culture medium immediately before use. For the animal experiments, plumbagin was dissolved in 5\% PEG 400 at the required concentrations.

Cell line and cell culture

The human breast cancer cell line MDA-MB-231SArfp was labeled with red fluorescent protein (RFP), which was separated from breast cancer bone metastatic sites and exhibited propensities of bone metastases and was kindly provided by Prof. Jiake Xu (University of Westem Australia, Australia) (19). The cell lines were generally inoculated in Dulbecco's Modified Eagle's medium (DMEM, HyClone, USA) supplemented with $10 \%$ fetal bovine serum (FBS) and $0.75 \mathrm{mg} \cdot \mathrm{mL}^{-1} \mathrm{G}-418$ for selection of cells that showed stable RFP expression (20). Cells were passaged at $37{ }^{\circ} \mathrm{C}$ in a humidified atmosphere with $5 \% \mathrm{CO}_{2}$.

\section{Animals}

Female $B A L B / C$ nude mice $(n=20)$ were obtained from the Shanghai Slac Laboratory Animal Company [Certificate No. SCXK (Shanghai) 2007-0005] at age 4-5 weeks and weighed approximately $20 \mathrm{~g}$. All of the animals were maintained under SPF conditions at $22.0{ }^{\circ} \mathrm{C} \pm 1{ }^{\circ} \mathrm{C}$ room temperature with a $12 \mathrm{~h}$ light/dark cycle and 40$60 \%$ humidity. All animals were unc onstrained regarding drinking and eating.

\section{Equipments}

The small animal non-invasive imaging system was implemented with an IVIS spectrum (Xenogen, Hopkinton, MA, USA). A digital X-ray camera wassupplied by Faxitron (MX-50 desktop X-ray radiograph).

\section{Transwell migration and invasion experiments}

A transwell method was used as previously reported (21). Briefly, 24-well transwell plates (8 $\mu$ m diameter, Corning, NY) were applied to measure migration. The basement membrane of the upper chamber was covered with Matrigel (BD Biosciences) at $0.3 \mu \mathrm{g} \cdot \mathrm{LL}^{-1}$ per well in invasion experiment. Breast cancer cells $\left(5 \times 10^{4}\right.$ per well) were treated with varied doses of plumbagin (noted as PL below) and suspended in serum-free DMEM added to the upper chamber. DMEM containing $10 \%$ FBS was 
added into the lower chamber as a chemoattractant and incubated at $37{ }^{\circ} \mathrm{C}$ for 24 hours. Cells that had failed to migrate were removed from the upper chamber with swabs; the remaining cells on the bottom side of the basement membrane were fixed with $4 \%$ paraformaldehyde for 10 minutes and stained with $1 \%$ crystal violet (dissolved in $2 \%$ ethanol) for 10 minutes. A random selection of 3-5 fields were photographed and counted under the microscope. All of the experiments were repeated at least 3 times.

\section{Real-time PCR a nalysis}

Twenty-four hours after treatment with different doses of PL on MDA-MB-231SArfp cells, RNA was extracted via a Trizol method to evaluate sample concentration and purity. A reverse transcription kit (iScript cDNA Synthesis
Kit, Bio-Rad) was used to obta in cDNA from the extracted RNA by the reverse transcription method. The mRNA expression levels of relevant factors were assessed using Real-Time PCR (Reagent Kit: SYBR@Premix Ex Taq ${ }^{\mathrm{TM}}$, Tii RNase H Plus, Takara) according to the manufacturer's instructions. Primers required for the experiment are shown in Table 1.

\section{Westem blot}

Whole cell extracts were prepared by lysing the cells in RIPA buffer (150 mmol. $\mathrm{L}^{-1} \mathrm{NaCl}, 1 \%$ sodium deoxycholate, $0.1 \%$ SDS, $50 \mathrm{mmol} \cdot \mathrm{L}^{-1}$ Tris- $\mathrm{HCl} \mathrm{pH} 7.4,1 \mathrm{mmol} \cdot \mathrm{L}^{-1}$ EDTA, $1 \mathrm{mmol} \cdot \mathrm{L}^{-1} \mathrm{PMSF}$, and $1 \%$ Triton $\left.\mathrm{X}-100\right)$, containing a cocktail of protease inhibitors and phosphatase inhibitors. Protein concentrations were detemined using the BCA protein assay (Thermo Sc ientific, IL, USA). A total of

Table 1 Primer sequences for Real-time PCR analysis

\begin{tabular}{|c|c|c|c|}
\hline Gene & Forward primer $\left(5^{\prime}-3^{\prime}\right)$ & Reverse primer $\left(5^{\prime}-3^{\prime}\right)$ & Product Length/bp \\
\hline IL-1a & AG ATG CCTG AG ATACCCAAAACC & CCAAGCACACCCAGTAGTCT & 147 \\
\hline IL-1 $\beta$ & ATG ATG G C TTATTACAG TG G CAA & GTCGGAGATTCGTAGCTGGA & 132 \\
\hline IL-6 & ACTCACCTCTICAG AACG AATTG & CCATC TITG GAAG GTTCAGG TTG & 149 \\
\hline IL-8 & ACTG AG AGTG ATTG AG AGTGGAC & AACCСTCTGCACCCAGTTIT & 112 \\
\hline TGF- $\beta$ & GGGACTATCCACCTGCAAGA & ССTCCTIGGCGTAGTAGTCG & 239 \\
\hline TNFa & ССТСТСТСТAATCAG СССТС TG & GAGGACCTGGGAGTAGATGAG & 220 \\
\hline MMP-2 & GATACCCCTITGACGGTAAGGA & ССТСTCССAAGGTCCATAGC & 112 \\
\hline MMP-9 & TTGACAGCGACAAGAAGTGG & GCCATTCACGTCGTCCTTAT & 179 \\
\hline GAPDH & CCTGCACCACCAACTGCTTA & AGGCCATGCCAGTGAGCTT & 178 \\
\hline
\end{tabular}

20-30 $\mathrm{\mu g}$ of protein was separated by SDS-PAGE and transferred to a nitrocellulose membrane. The proteins were detected using primary antibodies targeting $p$ STAT3, STAT3, or $\beta$-actin (Cell Signaling Technology, MA, USA). Protein bands were visualized using an Odyssey Infra red Imaging System (U-COR Biosc iences, Lincoln, NE USA).

\section{siRNA transfection}

MDA-MB-231SArfp cellswere seeded in 6-well platesat a density of $1 \times 10^{4}$ cells per well for $24 \mathrm{~h}$ to reach subconfluence. Next, cells were transfected with STAT3specific siRNA or a scrambled siRNA control (Santa Cruz Biotechnology) using Lipofectamine 2000 (Invitrogen, Carlsbad, CA) according to the manufacturer's instructions. The specific siRNAs consisted of pools of 3 targetspecific 20- to 25-nt siRNAs designed to knock down the target gene expression.

Construction of the breast cancerbone metastatic model and fluorescence detection

The exponentially growing cell line MDA-MB-231SArfp was digested with $2 \%$ trypsin. Cell suspension was produced by teminating digestion with DMEM culture medium, followed by re-suspending in PBS solution at 1000 r.min-1 for 4 min. Trypan Blue staining showed cell viability to be greater than $98 \%$. With PBS medium, cell concentration was adjusted to $5 \times 10^{6}$ per $\mathrm{mL}$. A suspension of $0.1 \mathrm{~mL}$ per nude mice was injected into the left ventric le of 20 female $B A L B / c$ mice and randomly assigned into two groups ( $n=10$ each group) with intraperitoneal administration of $100 \mu \mathrm{L} 5 \%$ PEG or $4 \mathrm{mg} \cdot \mathrm{kg}^{-1} \mathrm{PL} 5$ times a week for 7 weeks. Non-invasive fluorescence detection was measured once per week and recorded.

X-ray imaging and HE staining

$X$-ray imaging was used in tumor-loaded nude mice 7 weeks after injection of tumor cells. Metastatic tumors were removed and fixed with $4 \%$ paraformaldehyde for $24 \mathrm{~h}$, followed by a rinse of running water ovemight. Tissue sections, which were decalcified in 10\% EDTA at $4{ }^{\circ} \mathrm{C}$ for three weeks and embedded in paraffin, were made and observed by HE staining. 
Immunohistoc hemistry sta ining

Tissue sections were dewaxed and rehydrated, followed by an antigen retrieval process. Primary antibodies of MMP-2 and MMP-9 were added at $4{ }^{\circ} \mathrm{C}$ ovemight and inoculated with secondary antibodies after rinsing at room temperature for 1 hour. After DAB staining and microscopic observation, reactions were terminated followed by hematoxylin re-dyeing. Dehydration and xylene transparency were completed after differentiation by hydrochloride alcohol. The sections were sealed prior to microscopic observation. A selection of 3-5 random fields was photographed and counted by mic roscopy.

\section{Statistic al a nalysis}

All data were processed via SPSS 13.0 software. Quantita- tive materials were presented as $x \pm s$. Statistical correlation was evaluated by SPEARMAN level with $a=0.05$ as the bilateral signific ance level. A P-va lue of less than 0.05 wasconsidered to be statistic ally signific ant.

\section{Results}

PLattenuates migration and invasion of breast cancer cells

Inhibition of PL by 2.5-10 $\mu \mathrm{mol} \cdot \mathrm{L}^{-1}$ on MDA-MB-231SArfp cells was observed via a transwell method and showed a significant dose-related suppression of in vitro migration ability in MDA-MB-231SArfp cells (Figure 1A and 1C). Breast cancer cells harvested after pre-treatment of PL for $24 \mathrm{~h}$ were inoculated into a transwell chamber and covered with Matrigel gel for $24 \mathrm{~h}$. These results ind ic ate

\section{$\mathrm{PL} / \mu \mathrm{m} \quad 0$}

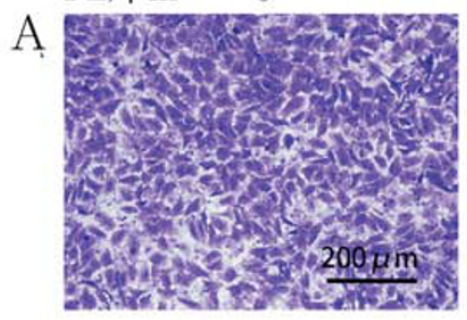

2.5

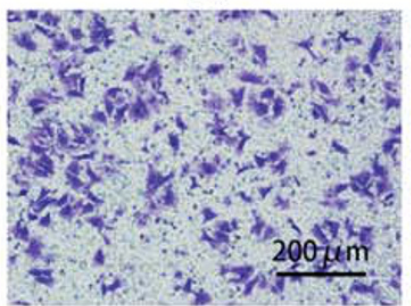

5

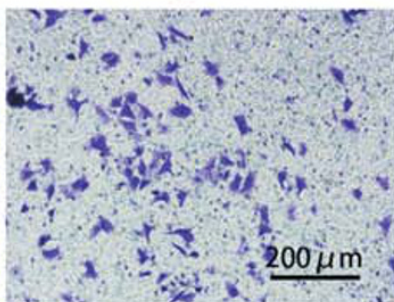

10

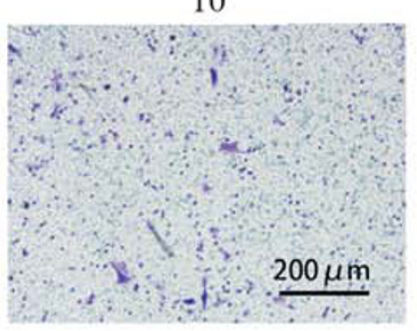

$\mathrm{PL} / \mu \mathrm{m} \quad 0$

$\mathrm{B}$

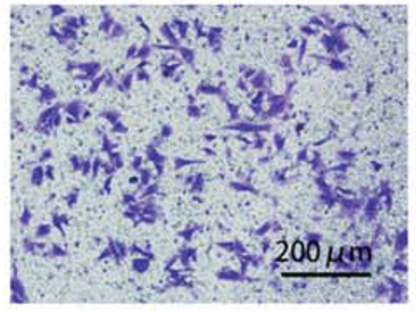

2.5

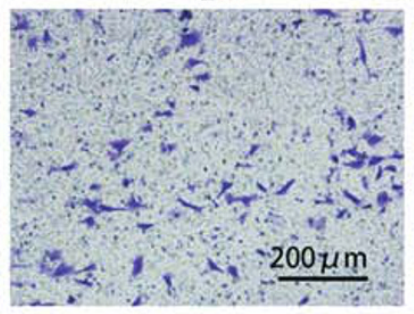

5

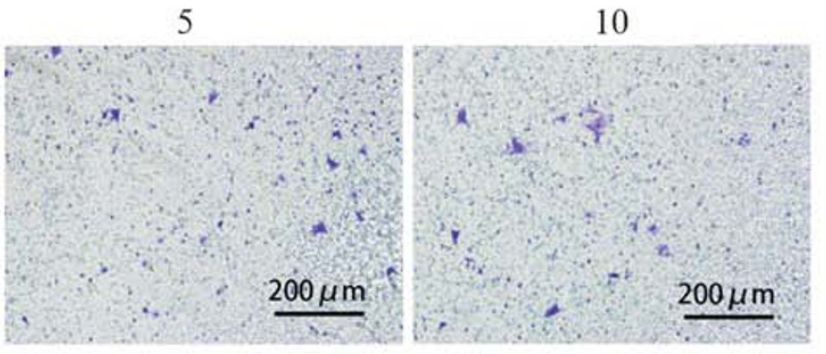

$\mathrm{D}$

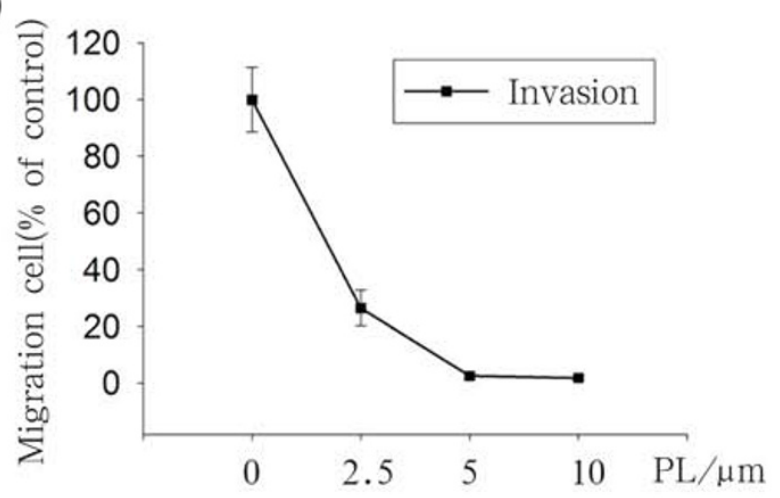

C

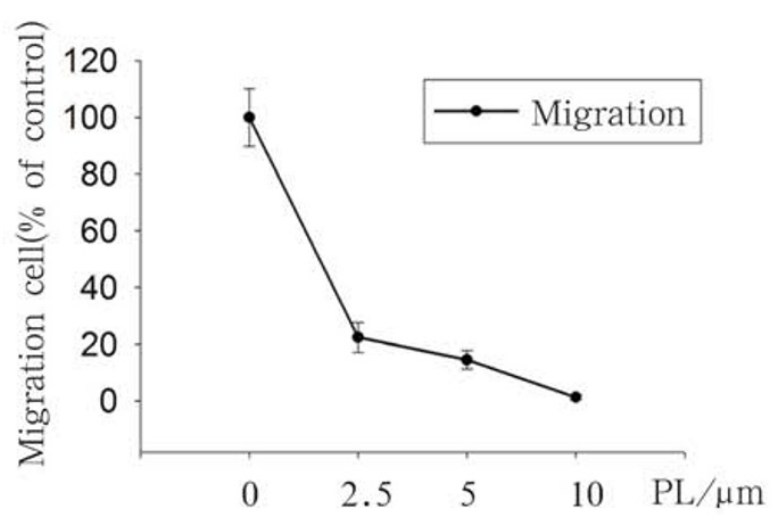

Figure 1 Effects of plumbagin on the invasion and migration of breast cancer cells. (A and C) Plumbagin (2.5-10 $\mu$ mol $\left.\cdot \mathrm{L}^{-1}\right)$ suppresses in vitro migration of MDA-SA-231SArfp cells at different doses. (B and D) Plumbagin $\left(2.5-10 \mu \mathrm{mol} \cdot \mathrm{L}^{-1}\right)$ decreases the invasion of MDA-SA-231SArfp cells and tumor cells show little penetration into matrigel at a concentration above $5 \mu \mathrm{mol} \cdot \mathrm{L}^{-1}$. 
that PL by $2.5-10 \mu \mathrm{mol} \cdot \mathrm{L}^{-1}$ is capable of markedly inhibiting invasion a bility of MDA-MB-231SArfp cells. Moreover, tumor cells could hardly penetrate matrigel gel to achieve the basement of chamber at a concentration more than $5 \mu \mathrm{mol} \cdot \mathrm{L}^{-1}$ (Figure 1B and 1D).

Effect of PL on mRNA expression levels of IL-1 $a, I L-1 \beta, I L-6$, IL-8, TGF- $\beta$, TNFa, MMP-2 and MMP-9 of breast cancer cells

RNA was extracted and then processed using a RealTime PCR reaction after treatment with $\mathrm{PL}$ at $5 \mu \mathrm{mol} \cdot \mathrm{L}^{-1}$ and $10 \mu \mathrm{mol} \cdot \mathrm{L}^{-1}$ on MDA-MB-231SArfp for $24 \mathrm{~h}$. The results indicate that, in comparison with the vehicle group (PL$\left.0 \mu \mathrm{mol} \cdot \mathrm{L}^{-1}\right)$, mRNA expressions of cytokine IL-1a and TGF- $\beta$, which are secreted from breast cancercells, decreased significantly. mRNA expressions of MMP-2 and MMP-9 from the matrix metalloproteinase family (when treated with $\left.\mathrm{PL}-10 \mu \mathrm{mol} \cdot \mathrm{L}^{-1}\right)$ were also greatly downregulated; however, mRNA expression of IL-6 dropped but exhibited no statistical significance. The remaining statistically significant factors were not observed in this research (Figure 2).

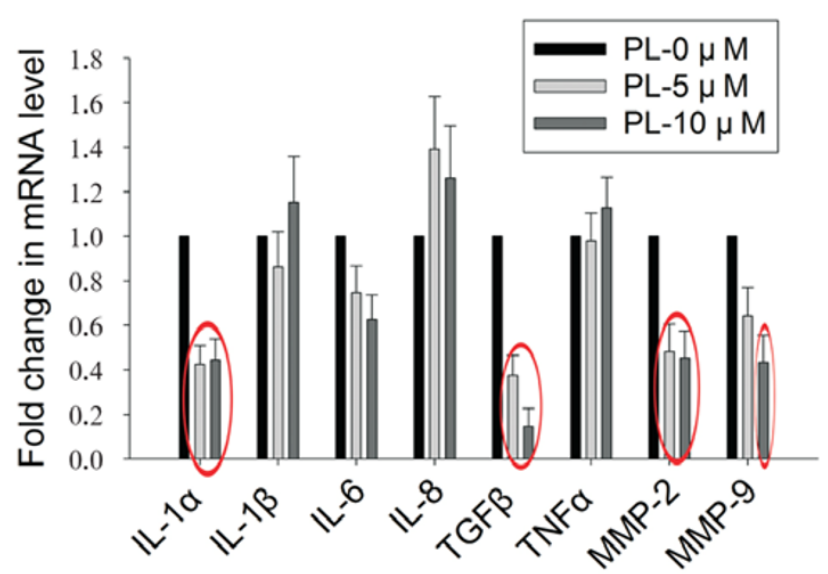

Figure 2 Effects of plumbagin on mRNA expressions of cytokines secreted from breast cancer cells. After treatment of plumbagin at $5 \mu \mathrm{mol} \cdot \mathrm{L}-1$ and $10 \mu \mathrm{mol} \cdot \mathrm{L}-1$ for $24 \mathrm{~h}$, mRNA expressions of IL-1a and TGF- $\beta$ secreted from MDA-SA-231SArfp as well as MMP-2 and MMP-9. mRNA expression of IL-6, however, showed a decrease without statistical significance.

PL inhibits the activation of STAT3 in breast cancercells To explore the mechanism whereby PL inhibited mRNA expression levels, MDA-MB-231SAifp cells were exposed to different concentrations of PL for 45 minutes. Westem blotting revealed that STAT3 activation was signific antly inhibited after 30 minutes (Figure 3A). Furthermore, we observed that PL inhibited activation of STAT3 in a dosedependent manner (Figure 3B). Subsequently, STAT3 in MDA-MB-231SArfp cells was knocked out by specific siRNA. Real-Time PCR results also demonstrated that the expression levels of IL-1 a, TGF- $\beta$, MMP-2 and MMP-9 were decreased (Figure $3 \mathrm{C}$ ).

PLattenuates metastases of breast cancer in nude mice Breast cancer cells were administered intracardially into nude mice followed by a gain in fluorescence signaling in the third week, thus demonstrating the existence of metastases. Few nude mice in PL treatment groups displayed weak fluorescence signals during the fourth week. The appearance of metastases and the intensity of fluorescence signal of systematic metastatic sites in the vehicle group, which was greater in number and intensity of metastatic sites of the PL treatment group, were observed in systematic skeletons and organs between the fourth and seventh weeks (Figure 4A and 4B).

PLameliorated osteolytic lesions caused by breast cancerbone metastases

$X$-ray imaging for eutha natized nude mice after 7 weeks demonstrated an increasing number of osteolytic lesions in the vehicle group, compared with a lower number and smaller area of bone lesions in the PL treatment group (Figure 5A). Knee joints of nude mice were selected for tissue sections, and HE staining showed obvious bone lesions in the vehicle group with a near complete disappearance of joint fomation, as well as few remaining sections of a rticular cartilage in the tibia. In contrast, intact articular structures and a smaller degree of destruction of bone tissues were observed in the PL treatment group, with smaller lumps of tumor tissue merely visible in the canal (Figure 5B).

The suppressive effects of PL on sec retions of MMP-2 and MMP-9 of breast cancercells

Tumor tissue was removed from euthanatized animals for fixation, paraffin embedding, tissue section and immunohistochemistry staining for MMP-2 and MMP-9. The results showed a lower number of MMP-2 stainingpositive breast cancer cells in the PL treatment group than the vehicle (as was shown in Figure 6A and 6B). Furthermore, tumor cells without treatment expressed high levels of MMP-9, which accounted for approximately $50 \%$ of all neoplasm in the cells. By contrast, MMP-9 staining-positive breast cancer cells in the PL treatment group decreased markedly (Figure 6A and 6C). 
A

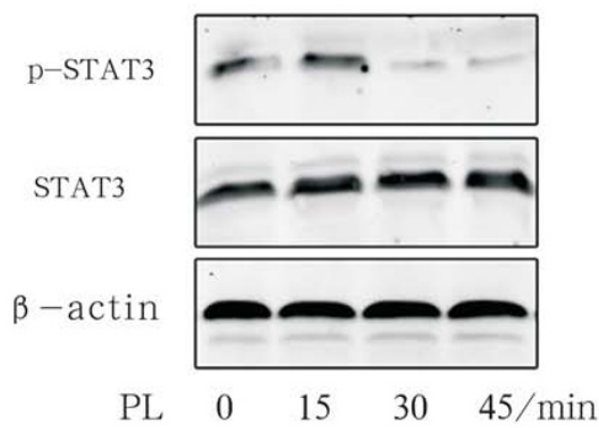

B

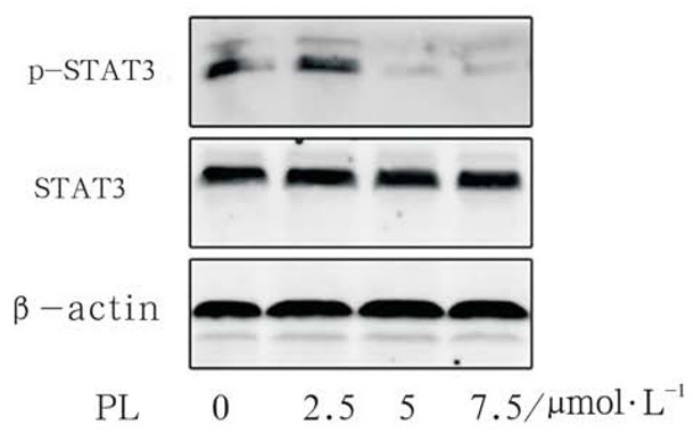

$\mathrm{C}$

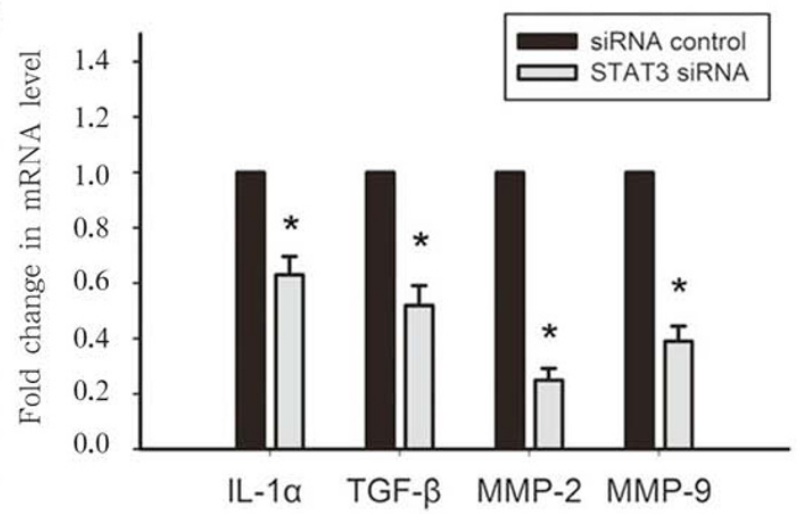

Figure 3 PL inhibits the activation of STAT3 in breast cancer cells. (A) MDA-SA-231SArfp cells were exposed to $7.5 \mu \mathrm{mol} \cdot \mathrm{L}^{-1} \mathrm{PL}$ for $45 \mathrm{~min}$ and STAT3 activation was detected by western blotting. (B) Western blot analysis of p-STAT3 and STAT3 protein levels in extracts from MDA-SA-231SArfp treated with PL for the indicated times. (C) MDA-SA-231SArfp was treated with STAT3 siRNA or control siRNA for $48 \mathrm{~h}$, and the expressions of IL-1a, TGF- $\beta$, MMP- 2 and MMP-9 mRNAs were detected by Real-Time PCR. The results are expressed as the mean \pm SD at ${ }^{*} P<0.05$.

A

$4 \mathrm{~W}$
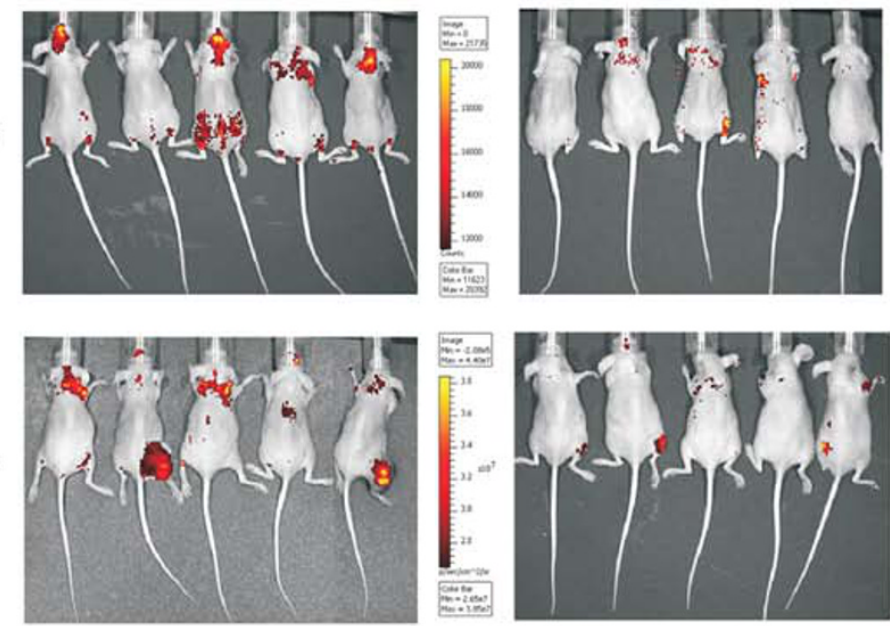

Vehicle
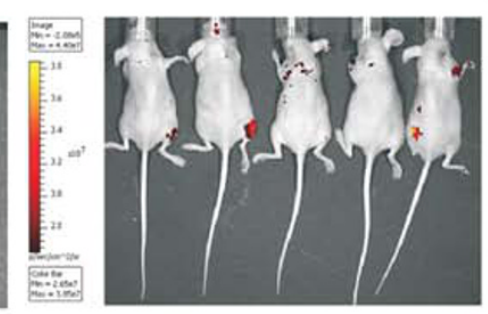

$\mathrm{PL}-4 \mathrm{mg}$

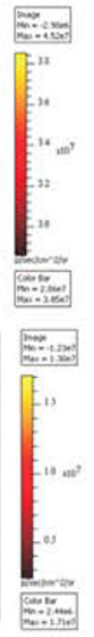

B

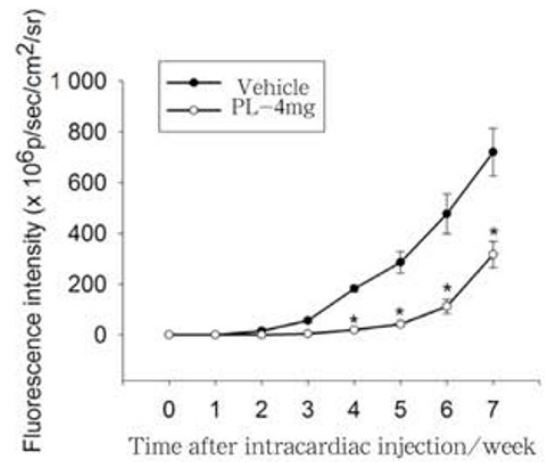

Figure 4 Effects of plumbagin on metastases of mammary cancer in nude mice. (A) Few mice exhibited weak fluorescence signals in the plumbagin treatment group during the fourth week. An increasing number of metastatic sites in systematic skeletons and organs were observed from the fourth to seventh weeks per vehicle group. However, in PL treatment cases, the number and intensity of fluorescence signals of metastatic sites were both less than the vehicle group. (B) Quantification of luminescence activities in the two groups at * $P<0.05$.

www.boneresearch.org | Bone Research 


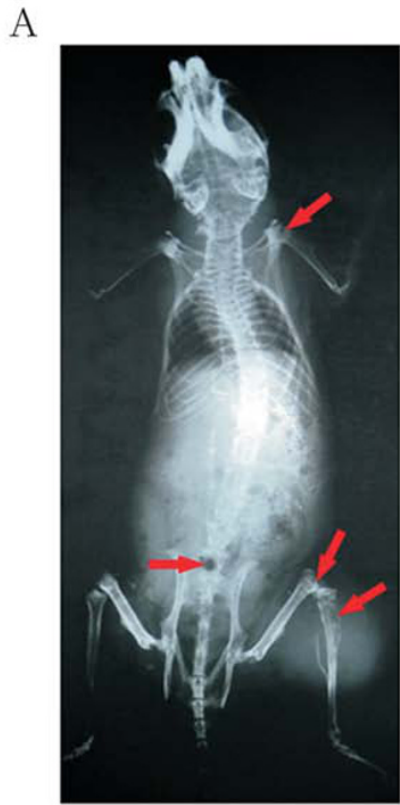

Vehicle

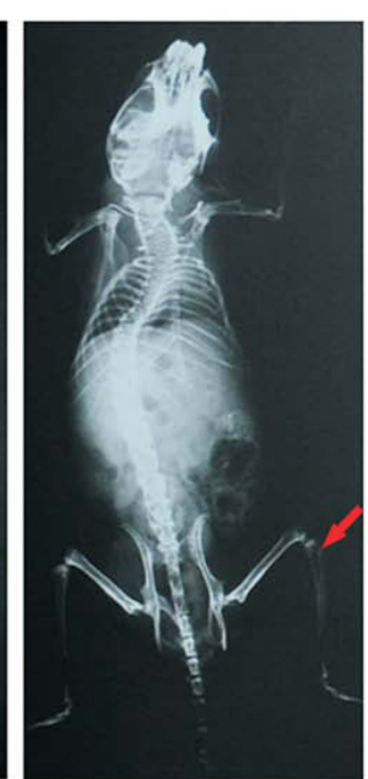

PL-4mg
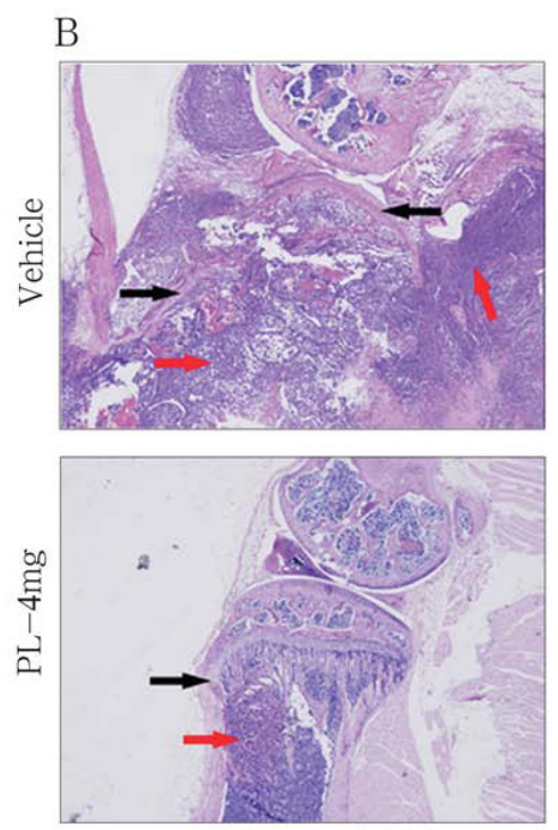

Figure 5 Effects of plumbagin on osteolytic lesions of breast cancer bone metastases. (A) Systematic skeleton X-ray imaging. In the vehicle group, a number of osteolytic lesions were observed in nude mice; in the PL treatment group, fewer osteolytic lesions were exhibited with lesion areas smaller than the vehicle. (B) HE staining of knee joints of nude mice. In the vehicle group, destruction of bone tissue in knee joints was obvious with the disappearance of joint structures and few remaining articular cartilage areas in the tibia. However, intact articular structure and fewer destruction of bone tissues were observed in the PL treatment group with small lumps of tumor tissue merely visible in the canal.

A
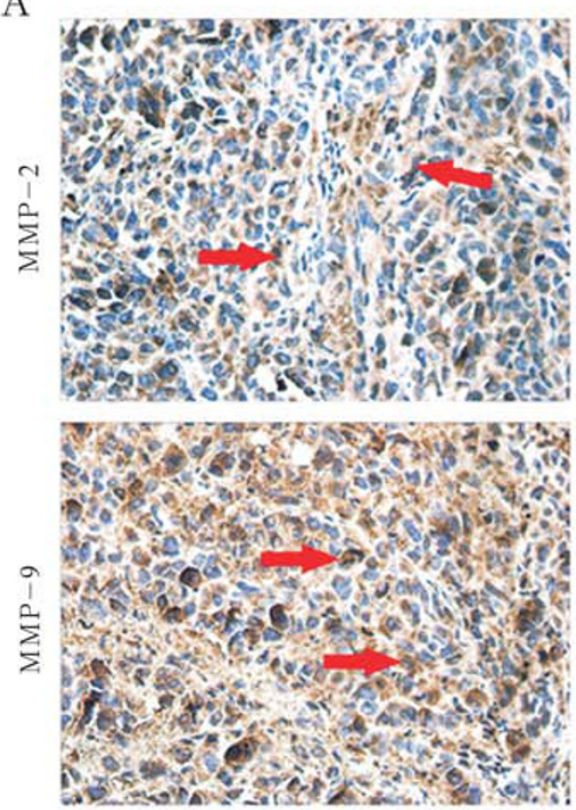

Vehicle
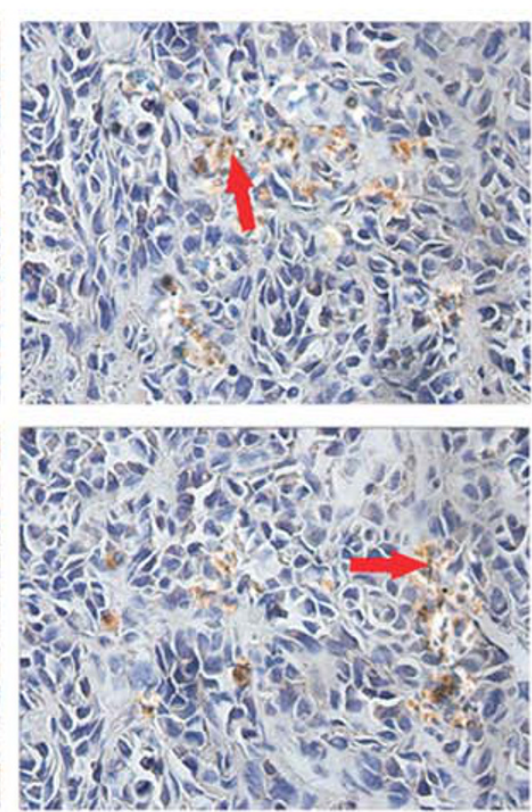

PL-4mg

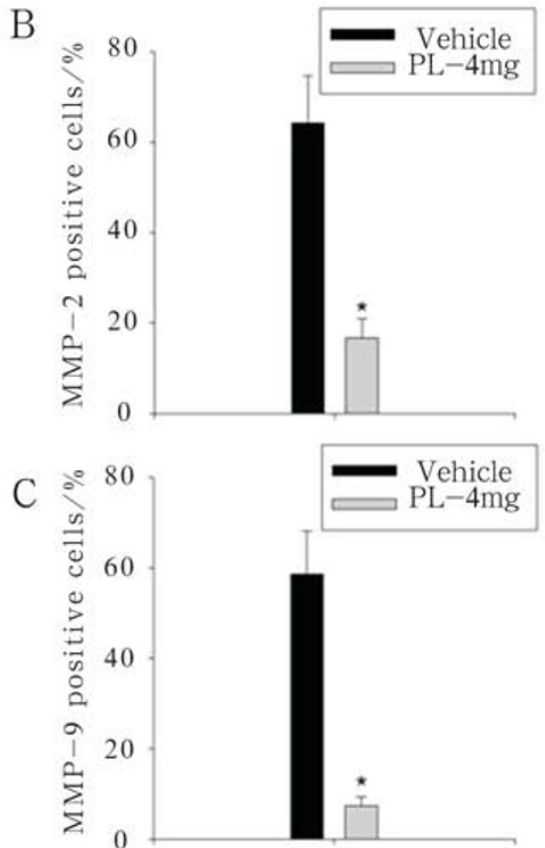

Figure 6 Effects of plumbagin on secretions of MMP-2 and MMP-9 in breast cancer cells. (A) immunohistochemistry staining, where fewer numbers of MMP-2 staining-positive breast cancer cells are shown in the PL treatment group than the vehicle. Tumor cells without treatment expressed high levels of MMP-9, which accounts for approximately 50\% of all tumor cells. By contrast, MMP-9 staining-positive breast cancer cells by PL treatment decreased markedly. (B-C) Quantification of MMP-2 and MMP-9 positive cells in the tumor tissues from the two groups. 


\section{Discussion}

Breast cancer bone metastases and resulting complications bring tremendous diffic ulties to clinical treatment, thus significantly impacting patients' quality of life and survival rates (22). In this study, we found that plumbagin could suppress migration and invasion of mammary neoplasm cells in vitro and reduce mRNA expressions of factors associated with migration and invasion of tumor cells. Plumbagin can inhibit distant dispersal of breast cancercells administered intracardia lly in vivo, especially bone metastases and osteolytic lesions. Additionally, MMP-2 and MMP-9 sec retion by tumor cells in metastatic sites is shown to be inhibited, and as a result the invasion ability of breast cancer cells is reduced.

In vitro, mRNA expression of IL-1 a, TGF- $\beta$, MMP-2 and MMP-9 by MDA-MB-231SArfp cells can be suppressed by plumbagin. Previous studies have shown that the binding between IL- 1 and IL- $1 R$ is considered to activate p38 MAPK and NF-KB signal transduction pathways, stimulating expression of VEGF and VEGF-2 and thus facilitating the growth of tumor cells (23). Moreover, recent studies have demonstrated that Leptin is capable of inducing proliferation and migration of breast carcinoma cells, and IL-1 plays a pivotal role in the signaling network (23) comprised of Leptin, IL-1 and Notch. Therefore, mammary neoplasm disease progression can likely be attenuated through decreased expression of IL-1. In breast cancer bone metastatic cycles, TGF- $\beta$ significantly enhances tumor development to release more PTHrP and thereby aggravates bone destruction (24). Recent studies have shown that curcuminoids can block TGF- $\beta$ signaling pathways to inhibit mammary cancer bone metastases in nude mice (25), which is similar to the mechanisms of plumbagin investigated in our study. MDA-MB-231SArfp cells treated with plumbagin for $24 \mathrm{~h}$, mRNA expression levels of MMP-2 and MMP-9 in the matrix metalloproteinases family was dec reased. Positive expression of MMP-2 and MMP-9 in tumor cells plays a vital role in their migration and invasion in vivo (26). MMP-9 is especially noted for decomposing the collagenous component of the ECM and devastating basic construction of collagen networks, thereby facilitating local invasion and distant migration (27).

STAT3 has been reportedly linked to tumor development and metastasis through induction of various genes responsible for cell proliferation, survival and carcinogenesis (28-30). In our experiment, we found that the activation of STAT3 was significantly inhibited when MDA-MB-231SArfp cells were exposed to different concentrations of $\mathrm{PL}$ after 30 minutes. Furthemore, we observed that the expressions IL-1a, TGF- $\beta$, MMP-2 and
MMP-9 were also decreased in MDA-MB-231SArfp cells while STAT3 was knocked out by specific siRNA. Thus, PL has potential antic ancer ac tivity aga inst breast cancer, which is likely due in part to inhibition of the STAT3 signaling pathway. Although we did not investigate the mechanisms of plumbagin-induced apoptosis in this study, previous results have reported the suppressive effects of plumbagin on DNA binding viability of NF-KB and $\mathrm{BCl}-2$, as well as induced apoptotic abilities against mammary cancer cells (31). Plumbag in is able to trigger a G2-M arrest and autophagy by inhibiting the AKT pathway in breast cancercells (13).

In conclusion, plumbagin is effective at attenuating the invasion and migration of breast cancer cells, decreasing distant tumor dispersal and reducing osteolytic lesions caused by bone metastases. As previous studies have also shown, future therapeutic applications of plumbagin are promising for the treatment of breast cancer bone metastases.

\section{Acknowledgments}

This work was supported by grants from the National Natural Science Foundation of China (No. 81172549), the Shanghai Science and Technology Development Fund (No. 11XD1403300), and the Opening Project of Shanghai Key Laboratory of Orthopaedic Implant (KFKT2011003), which are nonprofit foundations with no role in study design, data collection and analysis, decision to publish, or preparation of the manuscript.

\section{References}

1 Kamangar F, Dores GM, Anderson WF. Patterns of cancer incidence, mortality, and prevalence across five continents: defining priorities to reduce cancer disparities in different geographic regions of the world. J Clin Oncol. 2006;24: 2137-2150.

2 Mundy GR. Metastasis to bone: causes, consequences and therapeutic opportunities. Nat Rev Cancer. 2002;2:584-593.

3 Rose AA, Siegel PM. Breast cancer-derived factors facilitate osteolytic bone metastases. Bull Cancer. 2006;93:931-943.

4 Guise TA, Yin JJ, Taylor SD, Kumagai Y, Dallas M, Boyce BF, Yoneda T, Mundy GR. Evidence for a causal role of parathyroid hormone-related protein in the pathogenesis of human breast cancer-mediated osteolysis. J Clin Invest. 1996;98:1544-1549.

5 Mundy GR. Mechanisms of bone metastases. Cancer. 1997;80 1546-1556.

6 Lipton A, Theriault RL, Hortobagyi GN, Simeone J, Knight RD, Mellars K, Reitsma DJ, Heffernan M, Seaman JJ. Pamidronate prevents skeletal complications and is effective palliative treatment in women with breast carcinoma and osteolytic bone metastases. Cancer. 2000;88:1082-1090. 
7 Bamias A, Kastritis E, Bamia C, Moulopoulos LA, Melakopoulos I, Bozas G, Koutsoukou V, Gika D, Anagnostopoulos A, Papadimitriou C, Terpos E, Dimopoulos MA. Osteonecrosis of the jaw in cancer after treatment with bisphosphonates: incidence and risk factors. J Clin Oncol. 2005;23:8580-8587.

8 Durie BG, Katz M, Crowley J. Osteonecrosis of the jaw and bisphosphonates. N Engl J Med. 2005;353:99-102.

9 Dimopoulos MA, Kastritis E, Anagnostopoulos A, Melakopoulos I, Gika D, Moulopoulos LA, Bamia C, Terpos E, Tsionos K, Bamias A. Osteonecrosis of the jaw in patients with multiple myeloma treated with bisphosphonates: evidence of increased risk after treatment with zoledronic acid. Haematologica. 2006;91: 968-971.

10 Hoff AO, Toth BB, Altundag K, Johnson MM, Warneke CL, Hu M, Nooka A, Sayegh G, Guarneri V, Desrouleaux K, Cui J, Adamus A, Gagel RF, Hortobagyi GN. Frequency and risk factors associated with osteonecrosis of the jaw in cancer patients treated with intravenous bisphosphonates. J Bone Miner Res. 2008;23:826836.

11 de Paiva SR, Figueiredo MR, Aragão TV, Kaplan MA. Antimicrobial activity in vitro of plumbagin isolated from Plumbago species. Mem Inst Oswaldo Cruz. 2003;98:959-961.

12 Checker R, Sharma D, Sandur SK, Khanam S, Poduval TB. Anti-inflammatory effects of plumbagin are mediated by inhibition of NF-kappaB activation in lymphocytes. Int Immunopharmacol. 2009;9:949-958.

13 Kuo PL, Hsu YL, Cho CY. Plumbagin induces G2-M arrest and autophagy by inhibiting the $\mathrm{AKT} / \mathrm{mammalian}$ target of rapamycin pathway in breast cancer cells. Mol Cancer Ther. 2006;5: 3209-3221.

14 Aziz MH, Dreckschmidt NE, Verma AK. Plumbagin, a medicinal plant-derived naphthoquinone, is a novel inhibitor of the growth and invasion of hormone-refractory prostate cancer. Cancer Res. 2008;68:9024-9032.

15 Sand JM, Hafeez BB, Jamal MS, Witkowsky O, Siebers EM, Fischer J, Verma AK. Plumbagin (5-hydroxy-2-methyl-1, 4naphthoquinone), isolated from Plumbago zeylanica, inhibits ultraviolet radiation-induced development of squamous cell carcinomas. Carcinogenesis. 2012;33:184-190.

16 Sung B, Oyajobi B, Aggarwal BB. Plumbagin inhibits osteoclastogenesis and reduces human breast cancer-induced osteolytic bone metastasis in mice through suppression of RANKL signaling. Mol Cancer Ther. 2012;11:350-359.

17 Manu KA, Shanmugam MK, Rajendran P, Li F, Ramachandran L, Hay HS, Kannaiyan R, Swamy SN, Vali S, Kapoor S, Ramesh B, Bist P, Koay ES, Lim LH, Ahn KS, Kumar AP, Sethi G. Plumbagin inhibits invasion and migration of breast and gastric cancer cells by downregulating the expression of chemokine receptor CXCR4. Mol Cancer. 2011;10:107

18 Li Z, Xiao J, Wu X, Li W, Yang Z, Xie J, Xu L, Cai X, Lin Z, Guo W, Luo J, Liu M. Plumbagin inhibits breast tumor bone metastasis and osteolysis by modulating the tumor-bone microenvironment. Curr Mol Med. 2012;12:967-981
19 Yoneda T, Williams PJ, Hiraga T, Niewolna M, Nishimura R. A bone-seeking clone exhibits different biological properties from the MDA-MB-231 parental human breast cancer cells and a brainseeking clone in vivo and in vitro. J Bone Miner Res. 2001;16: 1486-1495.

20 Price JT, Quinn JM, Sims NA, Vieusseux J, Waldeck K, Docherty SE, Myers D, Nakamura A, Waltham MC, Gillespie MT, Thompson EW. The heat shock protein 90 inhibitor, 17-allylamino-17demethoxygeldanamycin, enhances osteoclast formation and potentiates bone metastases of a human breast cancer cell line. Cancer Res. 2005;21:4929-4938.

21 Tu B, Du L, Fan QM, Tang Z, Tang TT. STAT3 activation by IL-6 from mesenchymal stem cells promotes the proliferation and metastases of osteosarcoma. Cancer Lett. 2012;325:80-88.

22 Apte RN, Dotan S, Elkabets M, White MR, Reich E, Carmi Y, Song X, Dvozkin T, Krelin Y, Voronov E. The involvement of IL-1 in tumorigenesis, tumor invasiveness, metastasis and tumor-host interactions. Cancer Metastasis Rev. 2006;25:387-408.

23 Guo S, Gonzalez-Perez RR. Notch, IL-1 and leptin crosstalk outcome (NILCO) is critical for leptin-induced proliferation, migration and VEGF/VEGFR-2 expression in breast cancer. PloS one. 2011;6:e21467.

24 Baselga J, Rothenberg ML, Tabernero J, Seoane J, Daly T, Cleverly A, Berry B, Rhoades SK, Ray CA, Fill J. TGF- $\beta$ signalling-related markers in cancer patients with bone metastases. Biomarkers. 2008;13:217-236

25 Wright LE, Frye JB, Lukefahr AL, Timmermann BN, Mohammad KS, Guise TA, Funk JL. Curcuminoids block TGF-beta signaling in human breast cancer cells and limit osteolysis in a murine model of breast cancer bone metastasis. J Nat Prod. 2013;76: 316-321.

26 Fagan-Solis KD, Schneider SS, Pentecost BT, Bentley BA, Otis CN, Gierthy JF, Arcaro KF. The RhoA pathway mediates MMP-2 and MMP-9-independent invasive behavior in a triple-negative breast cancer cell line. J Cell Biochem. 2013;114:1385-1394.

27 Zhao S, Ma W, Zhang M, Tang D, Shi Q, Xu S, Zhang X, Liu Y, Song Y, Liu L, Zhang Q. High expression of CD147 and MMP-9 is correlated with poor prognosis of triple-negative breast cancer (TNBC) patients. Med Oncol. 2013;30:335.

28 Germain D, Frank DA. Targeting the cytoplasmic and nuclear functions of signal transducers and activators of transcription 3 for cancer therapy. Clin Cancer Res. 2007;13:5665-5669 .

29 Fukuda A, Wang SC, Morris JP, IVth, Folias AE, Liou A, Kim GE Akira S, Boucher KM, Firpo MA , Mulvihill SJ, Hebrok M. Stat3 and MMP7 contribute to pancreatic ductal adenocarcinoma initiation and progression. Cancer Cell. 2011;19:441-455.

30 Darnell JE. Validating Stat3 in cancer therapy. Nat Med. 2005;11 595-596.

31 Ahmad A, Banerjee S, Wang Z, Kong D, Sarkar FH. Plumbagininduced apoptosis of human breast cancer cells is mediated by inactivation of NF-kappaB and Bcl-2. J Cell Biochem. 2008;105: 1461-1471 